\title{
Ordinary Differential Equations and Integrable Models
}

\author{
Patrick Dorey, Clare Dunning, Roberto Tateo \\ SPhT Saclay, 91191 Gif-sur-Yvette, France (PED) \\ Dept. Math. Sciences, University of Durham, Durham DH1 3LE, UK (PED and TCD) \\ UVA, Inst. voor Theoretische Fysica, 1018 XE Amsterdam, The Netherlands (RT) \\ E-mails: p.e.dorey@dur.ac.uk, t.c.dunning@dur.ac.uk, tateo@wins.uva.nli
}

Abstract: We review a recently-discovered link between the functional relations approach to integrable quantum field theories and the properties of certain ordinary differential equations in the complex domain.

KEYworDs: 'Ordinary differential equations, spectral problems, Bethe ansatz, integrable models.

\section{Introduction}

Functional relations are assuming a growing importance in the study of integrable lattice models and integrable quantum field theories. The aim of this talk is to sketch a recently-discovered link between certain sets of these relations and a rather more classical area of mathematics, namely the theory of Stokes multipliers and spectral determinants for ordinary differential equations in the complex domain. For most of the talk the focus will be on the simplest example of this 'ODE/IM correspondence', connecting $2^{\text {nd }}$ order ordinary differential equations to Bethe ansatz systems of $S U(2)$ type. However, at the end some recent work extending this to $n^{\text {th }}$ order ODEs, and linking them to Bethe ansatz systems associated with $S U(n)$, will get a mention. To the extent that this talk has any logical structure at all, it is as follows:

(1) $2^{\text {nd }}$ order ODE (Schrödinger equations)

(2) Functional equations in integrable models

$\rightarrow(3)$ Connection

(4) Generalisations

Papers directly concerned with this topic include $[\overline{1}=[\overline{1}] \mid$, but it should be stressed it all relies heavily on earlier studies by, among others, Sibuya [1] $0_{1}^{\prime}$,

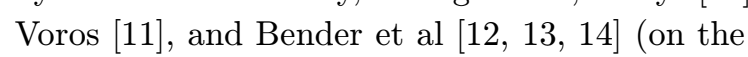

ODE side) and by Baxter [15ind, Klümper, Pearce

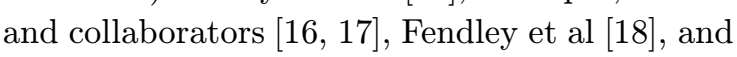
Bazhanov, Lukyanov and Zamolodchikov [1 $\left.19_{1}^{\prime},{ }^{2}{ }_{2}^{\prime \prime}\right]$ on the integrable models side.

\section{Schrödinger equations}

Stokes sectors, and their relationship with eigenvalue problems defined in the complex plane, will be important in the following, and to introduce these topics we begin by describing a class of problems much studied by Bender and collaborators in recent years. It all began with a question posed by Bessis and Zinn-Justin, many years ago...

Question 1: What does the spectrum of the Hamiltonian

$$
H=p^{2}+i x^{3}
$$

look like?

This is a cubic oscillator, with purely imaginary coupling $i$. (Strictly speaking, Bessis and ZinnJustin, motivated by considerations of the YangLee edge singularity, were interested in more general Hamiltonians of the form $p^{2}+x^{2}+i g x^{3}$, from which the above problem can be recovered as a strong-coupling limit.) The corresponding Schrödinger equation is

$$
-\frac{d^{2}}{d x^{2}} \psi(x)+i x^{3} \psi(x)=E \psi(x)
$$

and we will initially say that the (possibly complex) number $E$ will be in the spectrum if and 
only if, for that value of $E$, the equation has a solution $\psi(x)$ on the real axis which decays both at $x \rightarrow-\infty$ and at $x \rightarrow+\infty:^{1}$

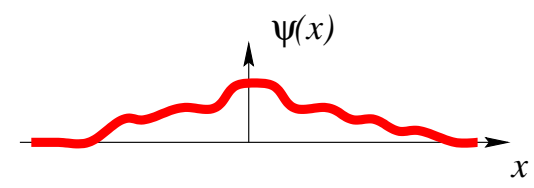

Note that the wavefunction $\psi(x)$ will inevitably be complex. Since the Hamiltonian is not (at least in any obvious way) Hermitian, the usual arguments to show that all of the eigenvalues $E$ must be real do not apply. Nevertheless, perturbative and numerical studies led Bessis and Zinn-Justin to the following conjecture:

- the spectrum of $H$ is real, and positive.

In 1997 Bender and Boettcher [1] ${ }_{1}^{1}$ in proposed a nice generalisation of this problem:

Question 2: What is the spectrum of

$$
H=p^{2}-(i x)^{N} \quad(N \text { real, }>0) ?
$$

Later, it will turn out that the passage from question 1 to question 2 corresponds to a change in a coupling constant in a sine-Gordon model, or of a quantum group deformation parameter in a Bethe ansatz system. But for now, the generalisation is appealing because it unites into a single family of eigenvalue problems both the $N=3$ case, for which we have the Bessis-Zinn-Justin conjecture, and the more easily-understood $N=2$ case, the harmonic oscillator. The Schrödinger equation is now

$$
-\frac{d^{2}}{d x^{2}} \psi(x)-(i x)^{N} \psi(x)=E \psi(x)
$$

and, as before, we look for those values of $E$ at which there is a solution along the real $x$-axis which decays at both plus and minus infinity. Two details need extra care: for non-integer values of $N$, the 'potential' $-(i x)^{N}$ is not singlevalued; and when $N$ hits 4 , the naive definition of the eigenvalue problem runs into difficulties. The first problem is easily cured by adding a branch cut along the positive imaginary $x$-axis, but the

\footnotetext{
${ }^{1}$ To be more precise, the decay should be fast enough that $\psi$ lies in $L^{2}(\mathbb{R})$, the space of square-integrable functions. This means that we are actually discussing the so-called point spectrum of $H$ - see, for example, [2네.
}

second is more subtle and will be discussed in greater detail below.

This caveat aside, there is already a surprise while $N$ remains below 4 . Figure $\left[A_{1}^{4}\right]$, and it reproduces the results of [1] $\left.{ }^{1} 3_{1}^{1}\right]$. Ignoring for a moment the region $N>4$, it is clear that something strange occurs as $N$ decreases through 2 - infinitely-many eigenvalues pair off and become complex, and only finitely-many remain real. By the time $N$ reaches 1.5 , all but three have become complex, and as $N$ tends to 1 the last real eigenvalue diverges to infinity. In fact, at $N=1$ the problem has no eigenvalues at all, as can be seen by solving the relevant Schrödinger equation in terms of an Airy function. For $N>2$, the numerically-obtained spectrum is entirely real, and positive, and so the conjecture of Bessis and Zinn-Justin has found a natural generalisation. The 'phase transition' to infinitely-many complex eigenvalues at $N=2$ was interpreted in [1 13 in as a spontaneous breaking of $\mathcal{P} \mathcal{T}$ symmetry.

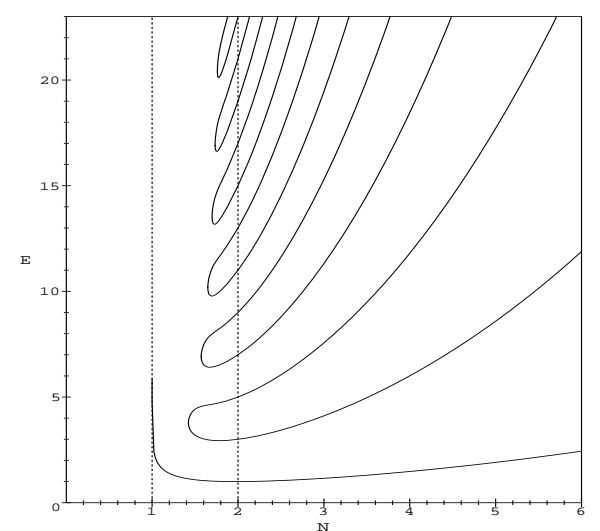

Figure $\overline{1}_{1}^{1}: H=p^{2}-(i x)^{N}$ : real eigenvalues as a function of $N$

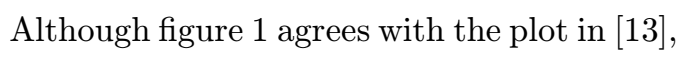
it was obtained in $[\mathbf{4}]$ by an entirely different route - rather than making a direct numerical attack on the ordinary differential equation, a nonlinear integral equation for the relevant spectral determinant was solved. This method of solving such eigenvalue problems is a byproduct of the ODE/IM correspondence and appears to be new, though it owes a heavy debt to earlier work of Voros [1] 1]. Numerically, it is rather efficient see for example the tables in [1] $\left[\begin{array}{l}1 \\ 1\end{array}\right]$ of eigenvalues of various anharmonic oscillators. 
Another idea motivated by the correspondence is the notion [iئin] to study the effect of an additional angular-momentum term $l(l+1) x^{-2}$ on the Bender-Boettcher problem. For $-1<l<0$, this turns out to have a remarkable effect on the behaviour of the spectrum as the $N=2$ phase transition is crossed.

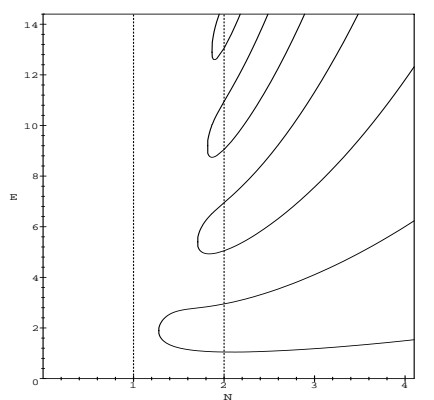

Figure 家: $H=p^{2}-(i x)^{N}-0.024735 x^{-2}$ : real eigenvalues as a function of $N$

Figure for $l=-0.025$, and reveals that the picture has changed dramatically - the connectivity of the real eigenvalues has been completely reversed, so that while for $l=0$ (the original Bender-Boettcher problem) the first and second excited states pair off, at $l=-0.025$ the first excited state is instead paired with the ground state, and so on up the spectrum. With this in mind, it may be a little hard to see how it is possible to pass between the sets of spectra depicted in figures by varying the continuous parameter $l$ from zero to -0.025 . The puzzled reader is invited to have

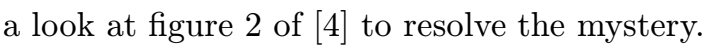

There remains one piece of unfinished business: what goes wrong at $N=4$, and what can be done to resolve it? On figures continue smoothly past $N=4$, but in fact this is only achieved by implementing a suitable distortion of the problem as originally posed. Consider the situation precisely at $N=4$ : the Hamiltonian is $p^{2}-x^{4}$, an 'upside-down' quartic oscillator, and a simple WKB analysis (about which more shortly) shows, instead of the exponential growth or decay more generally found, wavefunctions behaving as $x^{-1} \exp \left( \pm i x^{3} / 3\right)$ as $x$ tends to plus or minus infinity. All solutions thus decay, albeit algebraically, and this complicates matters significantly. The problem moves from what is called the limit-point to the limit-circle case (again, see [21in]), and additional boundary conditions should be imposed at infinity if the spectrum is to be discrete. While interesting in its own right, this is clearly not the right eigenproblem if we wish to find a smooth continuation from the region $N<4$. Instead, it is more fruitful to enlarge the perspective and treat $x$ as a genuinely complex variable. This has been discussed by many authors, and is particularly emphasised in the book by Sibuya [1 10 ; ; the treatment which follows is very close to that of [1 $12,1,13]$.

The key is to examine the behaviour of solutions as $|x| \rightarrow \infty$ along a general ray in the complex plane, even though the only two rays that we initially need are the positive and negative real axes. The WKB approximation tells us that

$$
\psi(x) \sim P(x)^{-1 / 4} e^{ \pm \int^{x} \sqrt{P(t)} d t}
$$

as $|x| \rightarrow \infty$, with $P(x)=-(i x)^{N}-E$. (This is easily derived by substituting $\psi(x)=f(x) e^{g(x)}$ into the ODE.) Since we set the problem up with a branch cut running up the positive-imaginary axis, it is natural to define a ray in the complex plane by setting $x=\rho e^{i \theta} / i$ with $\rho$ real:

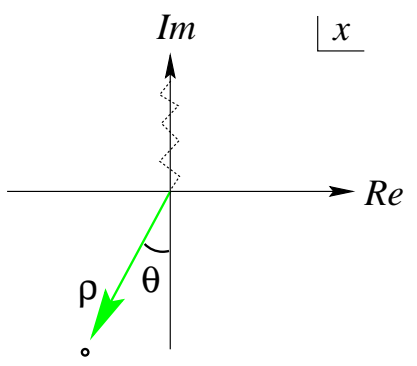

For $N>2$, the asymptotic is not changed if $P(x)$ is replaced by $-(i x)^{N}$, and substituting into the general formula we see two possible behaviours, as expected of a second-order ODE:

$$
\psi_{ \pm} \sim P^{-1 / 4} \exp \left[ \pm \frac{2}{N+2} e^{i \theta(1+N / 2)} \rho^{1+N / 2}\right] .
$$

For most values of $\theta$, one of these solutions will be exponentially growing, the other exponentially decaying. But whenever $\Re e\left[e^{i \theta(1+N / 2)}\right]=0$, the two solutions swap roles and there is a moment when both oscillate, and neither dominates the other. The relevant values of $\theta$ are

$$
\theta= \pm \frac{\pi}{N+2}, \pm \frac{3 \pi}{N+2}, \pm \frac{5 \pi}{N+2}, \ldots
$$


(Confusingly, the rays that these values of $\theta$ define are sometimes called 'anti-Stokes lines', and sometimes 'Stokes lines'.) Whenever one of these lines lies along the positive or negative real axis, the eigenvalue problem as originally stated becomes much more delicate, for the reasons described above. Increasing $N$ from 2, the first time that this happens is $N=4$, the case of the upside-down quartic potential. But now we see that the problem is easy to avert - it arose because the line along which the wavefunction was being considered, namely the real axis, happened to coincide with an anti-Stokes line ${ }^{2}$. But since all functions involved are analytic, there is nothing to stop us from examining the wavefunction along some other contour in the complex plane. In particular, before $N$ reaches 4 , the two ends of the contour can be bent downwards from the real axis without changing the spectrum, so long as their asymptotic directions do not cross any anti-Stokes lines in the process. Having thus distorted the original problem, $N$ can be increased through 4 without any difficulties. The situation for $N$ just bigger than 4 is illustrated below, with the anti-Stokes lines shown dashed and the wiggly line a curve along which the wavefunction $\psi(x)$ can be defined.

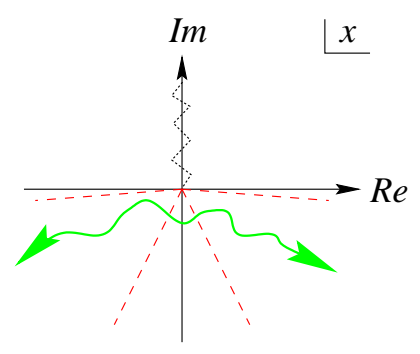

The wedges between the dashed lines are called Stokes sectors, and in directions out to infinity which lie inside these sectors, wavefunctions either grow or decay exponentially, leading to eigenvalue problems with straightforward, and discrete, spectra. Note that once $N$ has passed through 4 , as in the figure, the real axis is once again a 'good' quantisation contour - but for a different eigenvalue problem, which is not the analytic continuation of the original $N<4$ problem to that value of $N$. (For the analogue of figure for this new problem, see figure 20 of [1년].)

\footnotetext{
${ }^{2}$ as just mentioned, some would call this a Stokes line
}

There is a lesson to be drawn from all of

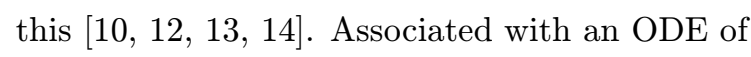
the type under consideration there are many different eigenvalue problems, each defined by specifying a pair of Stokes sectors, and then asking for the values of $E$ at which there exist solutions to the equation which decay exponentially in both simultaneously. For a given value of $N$, the two sectors which cover the positive and negative real axes may appear to be the most natural choice, but if we want to discuss analytic continuation then all must be put on an equal footing. This picture will find a precise analogue on the integrable models side of the correspondence, but before describing this we need to review some more basic material.

\section{Functional relations in integrable models}

In this section a very rough caricature of the 'functional relations' approach to integrable models will be given. A number of other speakers at the conference talked about this topic, in particular J.-M. Maillet, R. Poghossian and F. Smirnov, and their contributions should be consulted for more in-depth reviews. Not to forget, of course, the book [1] by Baxter...

We will discuss the six-vertex model, defined initially on an $N \times M$ lattice, with periodic boundary conditions and $N / 2$ even. On each (horizontal or vertical) link of the lattice, we place a spin 1 or 2 , conveniently depicted by an arrow pointing either left or right (for the horizontal links) or up or down (for the vertical links). Only those configurations of spins which preserve the 'flux' of arrows through each vertex are permitted. Locally this gives just six options (hence the name of the model) to which Boltzmann weights are assigned as follows:

$$
\begin{aligned}
& R_{11}^{11}=R_{22}^{22}=a \\
& R_{12}^{21}=R_{21}^{12}=b \\
& R_{12}^{12}=R_{21}^{21}=c
\end{aligned}
$$

The relative probability of finding any given configuration is found by multiplying together the Botzmann weights for the individual vertices, and 
a first quantity to calculate is just the sum of these numbers over all possible configurations the partition function, $Z$. Very crudely speaking, a model is said to be integrable if it is possible to evaluate quantities such as $Z$ (or even better, the free energy) exactly, at least in the limit where $N$ and $M$ both tend to infinity. The model under discussion turns out to be integrable in this sense for all values of $a, b$ and $c$. The overall normalisation factors out trivially from all calculations, and it is convenient to parametrise the remaining two degrees of freedom using a pair of variables $\nu$ (the spectral parameter) and $\eta$ (the anisotropy):

$a=\sinh (\nu-i \eta), b=\sinh (\nu+i \eta), c=\sinh (2 i \eta)$.

To calculate $Z$, one line of attack proceeds via the so-called transfer matrix, $T$ :

$$
T_{\alpha_{1} \alpha_{2} \ldots \alpha_{N}}^{\alpha_{1}^{\prime} \alpha_{2}^{\prime} \ldots \alpha_{N}^{\prime}}=\sum_{\left\{\beta_{i}\right\}} R_{\beta_{1} \alpha_{1}}^{\alpha_{1}^{\prime} \beta_{2}} R_{\beta_{2} \alpha_{2}}^{\alpha_{2}^{\prime} \beta_{3}} \ldots R_{\beta_{N} \alpha_{N}}^{\alpha_{N}^{\prime} \beta_{1}}
$$

The job of $T$, a $2^{N} \times 2^{N}$ matrix, is to perform the sum over a set of horizontal links. In this picture the indices of $T$ correspond to the spin variables sitting on the vertical links, which can now be summed by matrix multiplication. Thus:

$$
Z=\text { Trace }\left[T^{M}\right] \text {. }
$$

The next step is to compute via a diagonalisation of $T$. For example, the free energy per site in the limit $M \rightarrow \infty$ can be obtained as

$$
f=\frac{1}{N M} \log Z=\frac{1}{N M} \log \text { Trace }\left[T^{M}\right] \sim \frac{1}{N} \log t_{0}
$$

where $t_{0}$ is the largest eigenvalue of $T$ (corresponding to the ground state). Note that the eigenvalues $t_{0}, t_{1} \ldots$ are all functions of $\nu$ and $\eta$. However, there is still work to be done to find out what these functions are. At this point we just state that there exists a technique, the (algebraic) Bethe ansatz, for doing this. Skipping all details, the method works in two stages:

(i) Guess a form for an eigenvector of $T$, depending on a finite number of parameters $\nu_{1}, \ldots \nu_{n}$ (the 'roots').

(ii) Discover that this guess only works if the $\left\{\nu_{i}\right\}$ together solve a certain set of coupled equations (the 'Bethe ansatz equations').

These equations will be written down shortly, but first we describe a particularly neat reformulation that was found by Baxter. The first input is the fact that the transfer matrices commute at different values of $\nu$ :

$$
\left[T(\nu), T\left(\nu^{\prime}\right)\right]=0 .
$$

This means that they can be diagonalised simultaneously, with $\nu$-independent eigenvectors, and it allows us to focus on the individual eigenvalues $t_{0}(\nu), t_{1}(\nu), \ldots$ as functions of $\nu$. From the explicit form of the Boltzmann weights these functions are entire, and $i \pi$-periodic.

Now for the key result: for each eigenvalue function $t(\nu)$, there exists an auxiliary function $q(\nu)$, also entire and (at least for the ground state) $i \pi$-periodic, such that

$$
t(\nu) q(\nu)=a^{N} q(\nu+2 i \eta)+b^{N} q(\nu-2 i \eta) .
$$

We shall call this the T-Q relation, though this phrase might more properly be reserved for the corresponding matricial equation, involving $T(\nu)$ and another matrix $Q(\nu)$, from which the above can be extracted when acting on eigenvectors. It is not immediately clear why this result represents progress - we started with one unknown function $t(\nu)$, and have now been told that if we multiply this by another unknown function $q(\nu)$, we recover two copies of that same function at shifted values of its argument. However, the fact that both $t$ and $q$ are entire makes this condition much more restrictive than might first appear.

To make the match with the ODEs described in the last section, we will need to take a certain large- $N$ limit, simultaneously shifting $\nu$ and rescaling the TQ relation. This has the effect of eliminating the factors $a^{N}$ and $b^{N}$, and since it also simplifies the formulae, from here on we will assume that this has been done. The relation becomes

$$
t(\nu) q(\nu)=q(\nu+2 i \eta)+q(\nu-2 i \eta)
$$

and the $i \pi$-periodic function $q(\nu)$ can be written as a product over its zero positions as

$$
q(\nu)=\prod_{l} \sinh \left(\nu-\nu_{l}\right)
$$

Strictly speaking this product only converges if the number of zeroes is finite, which is not true in the limit we consider - the $\nu_{l}$ accumulate at infinity. Given certain growth conditions, $q(\nu)$ 
can more correctly be written as a product of factors $\left(1-e^{2\left(\nu-\nu_{l}\right)}\right)$. We are only interested in giving the flavour of the argument here, so having mentioned this caveat we retain the form appropriate for finite $N$. Now the reasoning goes as follows. First, we know from (TQ) that $t$ is fixed by $q$, and from (Q) that $q$ is fixed by the set $\left\{\nu_{i}\right\}$. To fix the $\left\{\nu_{i}\right\}$, set $\nu=\nu_{i}$ in (TQ). On the LHS we then have $t\left(\nu_{i}\right)$, which is nonsingular since $t$ is entire, times $q\left(\nu_{i}\right)$ which is zero by (Q). Thus the LHS vanishes, and rearranging we have

$$
\frac{q\left(\nu_{i}+2 i \eta\right)}{q\left(\nu_{i}-2 i \eta\right)}=-1, \quad i=1,2 \ldots
$$

or, using $(\mathrm{Q})$ one more time,

$$
\prod_{l} \frac{\sinh \left(\nu_{i}-\nu_{l}+2 i \eta\right)}{\sinh \left(\nu_{i}-\nu_{l}-2 i \eta\right)}=-1, \quad i=1,2 \ldots
$$

This is exactly the Bethe ansatz equation (BAE) for the problem, with the $\nu_{i}$ the roots. The formula for $t(\nu)$ implied by (TQ) then matches that resulting from a direct application of the algebraic Bethe ansatz. It is important to realise that the BAE does not have a unique solution, but a discrete set of them (infinite in the $N \rightarrow \infty$ limit), matching the fact $T$ has many eigenval$u^{3}{ }^{3}$. To select a particular solution, supplementary analyticity conditions should be imposed. In particular, the ground state emerges if we require that all of the $\nu_{i}$ lie on the real axis.

So far we have been discussing the behaviour of lattice models. However, Bazhanov, Lukyanov and Zamolodchikov were able to construct analogues of the $T$ and $Q$ operators directly in the context of a continuum quantum field theory [20 using a free-field representation of the massless limit of the sine-Gordon model. The functional relation (TQ) is then most usually written in terms of a variable $\lambda$, on which the 'shifts' on the RHS act multiplicatively, as follows:

$$
\begin{aligned}
& T(\lambda) A_{ \pm}(\lambda)= \\
& \quad e^{\mp 2 \pi i p} A_{ \pm}\left(q^{-1} \lambda\right)+e^{ \pm 2 \pi i p} A_{ \pm}(q \lambda)
\end{aligned}
$$

Here $T$ and $A_{ \pm}$are entire functions of $\lambda^{2}, q=$ $e^{i \pi \beta^{2}}$ with $\beta$ the sine-Gordon coupling, and $p$, an

\footnotetext{
${ }^{3}$ We won't go into the question of the completeness of the BAE solutions here; see [22] for a recent discussion.
}

extra parameter compared to the previous discussion, is related to the possibility of adding a twist to the periodic boundary conditions (an option which also exists on the lattice). Note also that $q$ can be interpreted as a quantum group deformation parameter.

\section{The TQ/ODE connection}

The goal now is to show that $\left(\mathrm{TQ}^{\prime}\right)$ also arises naturally in connection with the eigenvalue problems discussed in section 1. First, we need to develop our treatment of ordinary differential equations in the complex domain a little further, re-

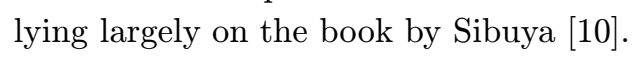

Consider the ODE

$$
\left[-\frac{d^{2}}{d x^{2}}+P(x)\right] \psi(x)=0
$$

where $P(x)=x^{2 M}-E$, and $M$ is real and positive. (This is the Bender-Boettcher problem with $N=2 M, x \rightarrow x / i$ and $E \rightarrow-E-$ a change which is made purely for convenience.) Then [23] the ODE $\left(^{*}\right)$ has a solution $y(x, E)$ such that

(i) $y$ is an entire function of $(x, E)$

[though $x$ lives on a cover of $\mathbb{C} \backslash\{0\}$ if $2 M \notin \mathbb{Z}$ ]

(ii) as $|x| \rightarrow \infty$ with $|\arg x|<3 \pi /(2 M+2)$,

$$
\begin{aligned}
y & \sim x^{-M / 2} \exp \left[-\frac{1}{M+1} x^{M+1}\right] \\
y^{\prime} & \sim-x^{M / 2} \exp \left[-\frac{1}{M+1} x^{M+1}\right]
\end{aligned}
$$

[though there are small modifications for $M \leq 1$ ] These properties fix $y$ uniquely; to understand where they come from we quickly recall the discussion of section 1 . With the shift from $x$ to $x / i$, the anti-Stokes lines for the current problem are

$$
\arg (x)= \pm \frac{\pi}{N+2}, \pm \frac{3 \pi}{N+2}, \ldots
$$

and in between them lie the Stokes sectors, which we label by defining

$$
\mathcal{S}_{k}=\left|\arg (x)-\frac{2 \pi k}{2 M+2}\right|<\frac{\pi}{2 M+2} .
$$

The asymptotic quoted in property (ii) is just the WKB result in $\mathcal{S}_{-1} \cup \mathcal{S}_{0} \cup \mathcal{S}_{1}$. One more piece of notation: an exponentially-growing solution in a given sector is called dominant (in that sector); 
one which decays is called subdominant. It is easy to check that $y$ as defined above is subdominant in $\mathcal{S}_{0}$, and dominant in $\mathcal{S}_{-1}$ and $\mathcal{S}_{1}$. Note that subdominant solutions to a second-order ODE are unique up to a constant multiple; this is why the quoted asymptotics pin down $y$ uniquely.

Having identified one solution to the ODE, we can now generate a whole family using a trick due to Sibuya. Consider the function $\hat{y}(x, E)=$ $y(a x, E)$ for some (fixed) $a \in \mathbb{C}$. From $(*)$,

$$
\left[-\frac{d^{2}}{d x^{2}}+a^{2 M+2} x^{2 M}-a^{2} E\right] \hat{y}(x, E)=0 .
$$

(This is sometimes given the rather-grand name of 'Symanzik rescaling'.) If $a^{2 M+2}=1$, it follows that $\hat{y}\left(x, a^{-2} E\right)$ solves $\left(^{*}\right)$. Setting

$$
\omega=e^{2 \pi i /(2 M+2)}
$$

and

$$
y_{k}(x, E)=\omega^{k / 2} y\left(\omega^{-k} x, \omega^{2 k} E\right)
$$

we therefore have the key statements

- $y_{k}$ solves $(*)$ for all $k \in \mathbb{Z}$;

- up to a constant, $y_{k}$ is the unique solution to $\left(^{*}\right)$ subdominant in $\mathcal{S}_{k}$. [This follows easily via the asymptotic of $y$.]

- each pair $\left\{y_{k}, y_{k+1}\right\}$ forms a basis of solutions for $(*)$. [This follows on comparing the asymptotics of $y_{k}$ and $y_{k+1}$ in either $\mathcal{S}_{k}$ or $\mathcal{S}_{k+1}$.]

We have almost arrived at the T-Q relation. First, expand $y_{-1}$ in the $\left\{y_{0}, y_{1}\right\}$ basis:

$$
y_{-1}(x, E)=C(E) y_{0}(x, E)+\widetilde{C}(E) y_{1}(x, E) .
$$

We will call this a Stokes relation, with the coefficients $C(E)$ and $\widetilde{C}(E)$ Stokes multipliers. They can be expressed in terms of Wronskians. A quick reminder [2] $\left.{ }^{4}\right]$ : the Wronskian of two functions $f$ and $g$ is

$$
W[f, g]=f g^{\prime}-f^{\prime} g .
$$

For two solutions of a second-order ODE with vanishing first-derivative term, $W[f, g]$ is independent of $x$, and vanishes if and only if $f$ and $g$ are proportional. To save ink we set

$$
W_{k_{1}, k_{2}}=W\left[y_{k_{1}}, y_{k_{2}}\right]
$$

and record the following two useful properties:

$$
W_{k_{1}+1, k_{2}+1}(E)=W_{k_{1}, k_{2}}\left(\omega^{2} E\right), \quad W_{0,1}(E)=2 i .
$$

Now by 'taking Wronskians' of the Stokes relation first with $y_{1}$ and then with $y_{0}$ we find

$$
C=\frac{W_{-1,1}}{W_{0,1}}, \quad \widetilde{C}=-\frac{W_{-1,0}}{W_{0,1}}=-1
$$

and so the relation can be rewritten as

$$
C(E) y_{0}(x, E)=y_{-1}(x, E)+y_{1}(x, E),
$$

or, in terms of the original function $y$, as

$$
\begin{aligned}
& C(E) y(x, E) \\
& =\omega^{-1 / 2} y\left(\omega x, \omega^{-2} E\right)+\omega^{1 / 2} y\left(\omega^{-1} x, \omega^{2} E\right) .
\end{aligned}
$$

This looks very like the T-Q relation! The only fly in the ointment is the $x$-dependence of the function $y$. But this is easily fixed: just set $x$ to zero. We can also take a derivative with respect to $x$ before setting it to zero, which swaps the phase factors $\omega^{ \pm 1 / 2}$. So we define

$$
D^{-}(E)=y(0, E) \quad, \quad D^{+}(E)=y^{\prime}(0, E) .
$$

(The notation will be justified shortly.) Then the Stokes relation implies

$$
\begin{aligned}
& C(E) D^{\mp}(E)= \\
& \omega^{\mp 1 / 2} D^{\mp}\left(\omega^{-2} E\right)+\omega^{ \pm 1 / 2} D^{\mp}\left(\omega^{2} E\right)
\end{aligned}
$$

Finally we are ready to make the comparison. If we set

$$
\beta^{2}=\frac{1}{M+1}, \quad p=\frac{1}{4 M+4}
$$

then the match between $\left(\mathrm{TQ}^{\prime}\right)$ and $(\mathrm{CD})$ is perfect, with the following correspondences between objects from the IM and ODE worlds:

$$
\begin{aligned}
T & \leftrightarrow C \\
A_{ \pm} & \leftrightarrow D_{\mp}
\end{aligned}
$$

How should we think about $C$ and $D$ ? In fact they are spectral determinants. Recall that $C(E)$ is proportional to $W_{-1,1}(E)$. Thus $C(E)$ vanishes if and only if $W\left[y_{-1}, y_{1}\right]=0$, in other words if and only if $E$ is such that $y_{-1}$ and $y_{1}$ are linearly dependent. But this means that $\left(^{*}\right)$ has a solution decaying in the two sectors $\mathcal{S}_{-1}$ and $\mathcal{S}_{1}$ simultaneously, which is exactly the spectral problem discussed in section 1 , modulo the trivial redefinitions of $x$ and $E$. This is enough to 
deduce that, up to a factor of an entire function with no zeroes, $C(E)$ is the spectral determinant for the Bender-Boettcher problem. Even this ambiguity can be eliminated, via Hadamard's factorisation theorem, once the growth properties of the functions involved have been checked; see [4] for details. To see that the functions $D^{ \pm}$are spectral determinants is even more easy: first, we note that by their very definition the functions $y(x, E)$ decay at (real) $x \rightarrow \infty$ for all values of $E$. If $D^{-}(E)=y(0, E)=0$, then this solution, decaying at $+\infty$, also vanishes at $x=0$, while if $D^{+}(E)=0$, then it has vanishing first derivative there. A moment's thought shows that this corresponds to there existing odd or even, respectively, wavefunctions for the equation on the full real axis with potential $|x|^{2 M}$. (It was for this reason that the functions $D^{ \pm}$were so labelled.)

This insight allows us to fill in one gap in the correspondence. While the $\mathrm{T}-\mathrm{Q}$ relation is very restrictive, as remarked in section 2 it does not have a unique solution. So to say that $D^{-}(E)$ is 'equal' to $A_{+}(\lambda)$ begs the question: which $A_{+}(\lambda)$ ? To answer, we first note that, in contrast to the Bender-Boettcher problem, the full-line problem with $|x|^{2 M}$ potential (or equivalently, the halfline problem with $y(0)=0$ boundary conditions) is self-adjoint, and so all of its eigenvalues are real. Back in the integrable model, the only solution to the BAE with all roots real is known to be the ground state, so the question is answered: the relevant $A_{+}(\lambda)$ is that corresponding to the ground state of the model.

As it stands, the correspondence is still not entirely satisfactory since, for each value of $\beta^{2}$, it picks out just one value of $p$. A more complete mapping would find partners for the BAE at other values of the twist parameter as well. This was sorted out very shortly after the original observation of the correspondence in 这: in Bazhanov, Lukyanov and Zamolodchikov pointed out that the ODE $(*)$ should be generalised to

$$
\left[-\frac{d^{2}}{d x^{2}}+x^{2 M}+\frac{l(l+1)}{x^{2}}-E\right] \psi(x)=0 .
$$

(This observation, combined with the discovery of the role of the $T$ operator made in [4i vided the motivation to study the spectra shown in figure $\overline{2}$, of section 1.) The previous mapping between parameters becomes

$$
\beta^{2}=\frac{1}{M+1}, \quad p=\frac{2 l+1}{4 M+4},
$$

and varying $l$ away from zero allows us to explore the other values of $p$. This is a continuation through continuous values of angular momentum in a radial (three-dimensional) Schrödinger equation - in other words, non-relativistic Regge theory! A little more care is needed in the definition of $D^{ \pm}$once $l(l+1)$ is nonzero, since the equation acquires a regular singularity at the origin. The resolution is to match the solutions $y_{k}$ onto solutions $\psi_{ \pm}$with simple scaling behaviours at the origin; the details can be found in $\left[\begin{array}{l}2 \\ 2\end{array} \overline{4} 1\right]$.

Two more points deserve a mention. First, studies of integrable models had already shown how to transform a T-Q relation into a nonlinear integral equation (NLIE), which in turn can be solved by numerical iteration rather easily [i $\left[\overline{7}_{1}\right.$, $2 \overline{5}^{\prime}, \overline{2}^{\prime} \overline{0}_{1}^{\prime}$. The NLIE is particularly simple for the ground state, and it was this that allowed the spectral plots of section 1 to be obtained in [i] with relatively little pain, building on the checks for specific cases performed in 1].1.1. Second, we should mention that there is another strand to the functional relations approach to integrable models, based on the so-called fusion hierarchy and its truncations (see for example $\left[\overline{1} \overline{1} \overline{6}_{n}^{\prime}, 1 \overline{1}, \overline{9} \mid\right]$ ). This proceeds via the definition of fused transfer matrices $T_{j}, j=0, \frac{1}{2}, 1, \frac{3}{2}, \ldots$ (with $T_{0}=1$ and the original $T$ identified with $T_{1 / 2}$ ), and ultimately leads to another set of nonlinear integral equations, often referred to as being of 'TBA type' $\left[{ }^{2} \overline{6}_{1}\right.$. Obviously it would be nice to find a role for these objects as well, and it turns out that this is possible. They are simply mapped onto the Wronskians $W_{k_{1}, k_{2}}$ with $\left|k_{1}-k_{2}\right|>2$ [4] and they therefore correspond to the other eigenvalue problems that were mentioned at the end of section 1 above. Truncation of the fusion hierarchy can then be reinterpreted in terms of the (quasi-)periodicity (in $k$ ) that the functions $y_{k}$ exhibit whenever $M$ is rational. In the simplest cases (with $M$ rational and $l(l+1)=0$ ) this periodicity arises because the solutions to the ODE live on a finite cover of $\mathbb{C} \backslash\{0\}$; for other cases, the monodromy around $x=0$ needs a little more care, but the story remains essentially the same. 
All good correspondences need a dictionary, and to end this section we give a summary of the mapping between objects seen by the integrable model and the Schrödinger equation:

\begin{tabular}{|c|c|c|}
\hline $\begin{array}{l}\text { Integrable } \\
\text { Model }\end{array}$ & & $\begin{array}{l}\text { Schrödinger } \\
\text { equation }\end{array}$ \\
\hline Spectral parameter & $\leftrightarrow$ & Energy \\
\hline Anisotropy & $\leftrightarrow$ & Degree of potential \\
\hline Twist parameter & $\leftrightarrow$ & Angular momentum \\
\hline $\begin{array}{l}\text { (Fused) transfer } \\
\text { matrices }\end{array}$ & $\leftrightarrow$ & $\begin{array}{l}\text { Spectral problems de- } \\
\text { fined at }|x|=\infty\end{array}$ \\
\hline Q operators & $\leftrightarrow$ & $\begin{array}{l}\text { Spectral problems link- } \\
\text { ing }|x|=\infty \text { and }|x|=0\end{array}$ \\
\hline $\begin{array}{l}\text { Truncation of the } \\
\text { fusion hierarchy }\end{array}$ & $\leftrightarrow$ & $\begin{array}{l}\text { Solutions on finite cov- } \\
\text { ers of } \mathbb{C} \backslash\{0\}\end{array}$ \\
\hline
\end{tabular}

(The two classes of spectral problems mentioned in this table are related to the 'lateral connection' and 'radial connection' problems in general WKB theory - see, for example, [2근.

Armed with the dictionary, the horizontal axis of figure integrable models correspond to the various values of $N$ in the Bender-Boettcher problem. Thus for $N=1,2,3,4$ and 6 , the relevant integrable models are the $N=2$ SUSY point of the sineGordon model, the free-fermion point, the YangLee model, $\mathbb{Z}_{4}$ parafermions and the 4 -state Potts model respectively. It is amusing that the $x^{3}$ potential is related by the correspondence to the Yang-Lee model (or, strictly speaking, to the sineGordon model at the value of the coupling which allows for a reduction to Yang-Lee), thus returning by a very indirect route to a neighbourhood of the original thought of Bessis and Zinn-Justin.

\section{Generalisations}

The Bethe ansatz equations seen so far can all be written in terms of the variable $E$ as

$$
\prod_{j=1}^{\infty}\left(\frac{E_{j}-\omega^{2 M} E_{k}}{E_{j}-\omega^{-2 M} E_{k}}\right)=-\omega^{2 l+1}, \quad k=1,2 \ldots
$$

where $\omega=e^{2 \pi i /(2 M+2)}, M$ is related to the quantum group deformation parameter, or anisotropy, of the lattice model, and $l$ is related to the twist.
These are the $n=2$ cases of a general family of $S U(n)$-related Bethe ansatz systems, relating $n-1$ sets of unknowns $\left\{E_{k}^{(m)}\right\}$, with $m=$ $1,2 \ldots n-1$ and $k=1,2 \ldots \infty$ :

$$
\prod_{t=1}^{n-1} \prod_{j=1}^{\infty}\left(\frac{E_{j}^{(t)}-\omega^{\frac{n M}{2} C_{m t}} E_{k}^{(m)}}{E_{j}^{(t)}-\omega^{-\frac{n M}{2} C_{m t}} E_{k}^{(m)}}\right)=-\omega^{n \tau_{m}+1} .
$$

As in the $S U(2)$ case, $M$ can be viewed as a deformation parameter, but this time there are not one but $n-1$ independent twists, $\tau_{1}, \tau_{2}, \ldots \tau_{n-1}$. The indices $m$ and $t$ should be thought of as living on an $S U(n)$ Dynkin diagram, of which $C_{m t}$ is the Cartan matrix. To obtain these equations using operators defined directly in a continuum quantum field theory, as achieved in [1] $\left.{ }^{1} \underline{g}_{1}^{1},{ }_{2}^{2} \mathbf{L}_{1}^{\prime}\right]$ for the $S U(2)$ case, appears to be a largely open problem, though the first steps have been undertaken in [28] ]. But even without this motivation, it is very natural to ask whether the correspondence described above can be extended to cover BA systems of these more general types.

The answer is yes [i, and it turns out that one has to turn to higher-order ordinary differential equations. Earlier but less complete results

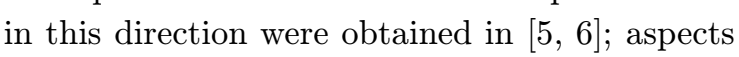
of the problem are also discussed in the recent

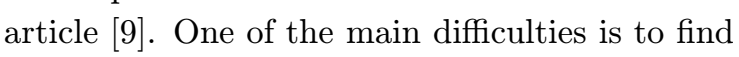
a parametrisation of the higher-order differential operators which incorporates the twists in a manageable way. The solution found in [8] starts by defining an elementary first-order differential operator, $D(g)$ :

$$
D(g)=\left(\frac{d}{d x}-\frac{g}{x}\right) .
$$

Elementary properties are $D(g)^{\dagger}=-D(-g)$ and $D\left(g_{2}-1\right) D\left(g_{1}\right)=D\left(g_{1}-1\right) D\left(g_{2}\right)$. Now, given a vector $\mathbf{g}=\left(g_{0}, g_{1} \ldots g_{n-1}\right)$, set

$$
\begin{aligned}
& D(\mathbf{g})= \\
& \quad D\left(g_{n-1}-(n-1)\right) D\left(g_{n-2}-(n-2)\right) \ldots D\left(g_{0}\right)
\end{aligned}
$$

and impose $\sum_{i=0}^{n-1} g_{i}=n(n-1) / 2$ to ensure that the $(n-1)^{\text {th }}$ order derivative term vanishes (this allows various theorems about Wronskians to hold in their simplest forms). With this notation in place, the ODE to consider is an immediate generalisation of those seen earlier:

$$
\left((-1)^{n+1} D(\mathbf{g})+P(x, E)\right) \psi(x)=0
$$


with $P(x, E)=x^{n M}-E$. After some work, it turns out this ODE does indeed contain a hidden set of $S U(n)$ Bethe ansatz equations. The parameter $M$ in $P(x, E)$ is equal to the $M$ appearing in the $S U(n)$ BAE quoted above, while the vector of parameters $\mathbf{g}$ is related to the twists in the BAE by

$\mathbf{g}=\left(\begin{array}{c}0 \\ 1 \\ 2 \\ \vdots \\ n-1\end{array}\right)+\left(\begin{array}{ccccc}1-n & 2-n & 3-n & \ldots \\ 1 & 2-n & 3-n & \ldots \\ 1 & 2 & 3-n & \ldots \\ \vdots & \vdots & \vdots & \\ 1 & 2 & 3 & \ldots\end{array}\right)\left(\begin{array}{c}\tau_{1} \\ \tau_{2} \\ \vdots \\ \tau_{n-1}\end{array}\right)$

The $S U(n)$ structure is encoded via certain Wronskians $W\left[y_{i}\right], W\left[y_{i}, y_{j}\right], W\left[y_{i}, y_{j}, y_{k}\right] \ldots$ These are $m$-dimensional determinants of matrices formed by the functions $y_{k_{1}}, \ldots y_{k_{m}}$ and their first $(m-1)$ derivatives, for $m=1 \ldots n-1$. The functions $y_{k}$ themselves are certain special solutions of the ODE, subdominant in particular sectors of the complex plane. They generalise the $y_{k}$ introduced by Sibuya for second-order ODEs, that were described in section 3 above. For the precise definitions and more details of how the mapping goes, the paper [i] should be consulted, since space prevents a fuller discussion in this short review.

\section{Conclusions}

The headline conclusion of this talk should already be clear: it is that the $T$ and $Q$ operators which arise in certain integrable quantum field theories encode spectral data, at least in their ground-state eigenvalues. This gives a novel perspective on the Bethe ansatz, and also a new way to treat spectral problems via the solution of nonlinear integral equations.

One important topic not covered here is the new light that the correspondence sheds on some previously-conjectured duality properties of inte-

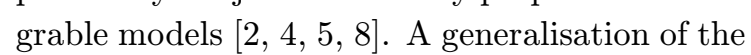
Langer [2$\overline{2} \overline{9}]$ transformation can be employed to map the ODE with the potential $x^{M}$ to one with potential $x^{\widetilde{M}}, \widetilde{M}=-M /(M+1)$. For $n=2$, this sends the parameter $q=e^{i \pi /(M+1)}$ to $\widetilde{q}=$ $e^{i \pi /(\widetilde{M}+1)}=e^{i \pi(M+1)}$, which is precisely the kind of duality discussed in the talk by F. Smirnov at this conference.
There are many further problems to be explored, of which we list just a few. First, one would like to know how many other BA systems can be brought into the correspondence, beyond the $A_{n-1}$-related cases described above, and whether more general polynomial potentials might also have a role to play. The set is certainly not empty - see $[\overline{7} \overline{1}-$ - but the problem of finding ODEs even for the $D$ and $E$ related BA systems remains open. Second, the correspondences established to date have all concerned massless integrable lattice models, in a 'field theory' limit where the number of sites, and of Bethe ansatz roots, tends to infinity. Correspondences for more general massive models, and for lattice models with a finite number of sites, would be very interesting. Finally, we should admit that our observations remain at a rather formal and mathematical level. At some stage one should ask what physics lies behind all of this, but perhaps such questions will have to wait until the answers to the other open problems have been found and classified. In this sense, we may still be in a 'stamp-collecting' phase, and we can expect that further work will lead to a much more systematic understanding of the whole story.

\section{Acknowledgments}

We would like to thank Junji Suzuki and André Voros for their helpful comments. PED thanks the organizers of the TMR2000 meeting for the invitation to speak. We were supported in part by a TMR grant of the European Commission, reference ERBFMRXCT960012.

\section{References}

[1] P. Dorey and R. Tateo, 'Anharmonic oscillators, the thermodynamic Bethe ansatz and nonlinear integral equations', J. Phys. A32 (1999) L419, hep-th/9812211'

[2] V.V. Bazhanov, S.L. Lukyanov and A.B. Zamolodchikov, 'Spectral determinants for Schrödinger equation and Q-operators of Conformal Field Theory', hep-th/9812247,

[3] J. Suzuki, 'Anharmonic Oscillators, Spectral Determinant and Short Exact Sequence of $\underline{U}_{q}\left(\widehat{s}_{2}\right)_{2}^{\prime}$, J. Phys. A32 (1999) L183, hep-th/9902053 
[4] P. Dorey and R. Tateo, 'On the relation between Stokes multipliers and the T-Q system of conformal field theory',_Nucl. Phys. B563 (1999) 573, hep-th/9906219,

[5] P. Dorey and R. Tateo, 'Differential equations and integrable models: the $S U(3)$ case', Nucl. Phys. B571 (2000) 583, hep-th/9910102'

[6] J. Suzuki, 'Functional relations in Stokes multipliers and Solvable Models related to $U_{q}\left(A_{n}^{(1)}\right)^{\prime}$, J. Phys. A33 (2000) 3507, hep-th/9910215

[7] J. Suzuki, 'Functional relations in Stokes multipliers - Fun with $x^{6}+\alpha x^{2}$ potential', quant-ph/0003066,

[8] P. Dorey, C. Dunning and R. Tateo, 'Differential equations for general $S U(n)$ Bethe ansatz systems', hep-th/0008039'

[9] J. Suzuki, 'Stokes multipliers, Spectral Determinants and T-Q relations', nlin-sys/0009006

[10] Y. Sibuya, Global Theory of a second-order linear ordinary differential equation with polynomial coefficient (Amsterdam: North-Holland 1975)

[11] A. Voros, 'Semi-classical correspondence and exact results: the case of the spectra of homogeneous Schrödinger operators', J. Physique Lett. 43 (1982) L1;

- 'The return of the quartic oscillator. The complex WKB method', Ann. Inst. Henri Poincaré Vol XXXIX (1983) 211;

- 'Exact resolution method for general 1D polynomial Schrödinger equation', J. Phys. A32 (1999) 5993, math-ph/9903045

[12] C.M. Bender and A. Turbiner, 'Analytic continuation of eigenvalue problems', Phys. Lett. A173 (1993) 442

[13] C.M. Bender and S. Boettcher, 'Real spectra in non-hermitian Hamiltonians having $\mathcal{P} \mathcal{T}$ symmetry', Phys. Rev. Lett. 80 (1998) 4243, physics/9712001

[14] C.M. Bender, S. Boettcher and P.N. Meissinger, ' $\mathcal{P} \mathcal{T}$ symmetric quantum mechanics', J. Math. Phys. 40 (1999) 2201, quant-ph/9809072

[15] R. Baxter, Exactly solved models in statistical mechanics (Academic Press 1982)

[16] A. Klümper and P.A. Pearce, 'Analytical calculations of Scaling Dimensions: Tricritical
Hard Square and Critical Hard Hexagons', J. Stat. Phys. 64 (1991) 13

[17] A. Klümper, M.T. Batchelor and P.A. Pearce, 'Central charges of the 6- and 19-vertex models with twisted boundary conditions', J. Phys. A24 (1991) 3111

[18] P. Fendley, F. Lesage and H. Saleur, ' Solving 1-D plasmas and 2-D boundary problems using Jack polynomials and functional relations', J. Stat. Phys. 79 (1995) 799, hep-th/94091766. - 'A unified framework for the Kondo problem and for an impurity in a Luttinger liquid', J. Stat. Phys. 85 (1996) 211, 'cond-mat/9510055'

[19] V.V. Bazhanov, S.L. Lukyanov and A.B. Zamolodchikov, 'Integrable Structure of Conformal Field Theory, Quantum KdV Theory and Thermodynamic Bethe Ansatz', Commun. Math. Phys. 177 (1996) 381, hep-th/9412229,

[20] V.V. Bazhanov, S.L. Lukyanov and A.B. Zamolodchikov, 'Integrable structure of conformal field theory II. Q-operator and DDV equation', Commun. Math. Phys. 190 (1997) 247, hep-th/9604044'

[21] M. Reed and B. Simon, Methods of Modern Mathematical Physics, I 6 II (Academic Press 1972, 1975);

R.D. Richtmyer, Principles of Advanced Mathematical Physics I (Spinger-Verlag 1978)

[22] K. Fabricius and B.M. McCoy, 'Bethe's equation is incomplete for the $\mathrm{XXZ}$ model at roots of unity', 'cond-mat/0009279,

[23] P.-F. Hsieh and Y. Sibuya, 'On the asymptotic integration of second order linear ordinary differential equations with polynomial coefficients', J. Math. Analysis and Applications 16 (1966) 84

[24] Earl A. Coddington and Norman Levinson, Theory of Ordinary Differential Equations, McGraw-Hill 1955

[25] C. Destri and H.J. de Vega, 'New thermodynamic Bethe ansatz equations without strings', Phys. Rev. Lett. 69 (1992) 2313 ;

- 'Unified approach to thermodynamic Bethe ansatz and finite size corrections for lattice models and field theories', Nucl. Phys. B438 (1995) 413

[26] Al.B. Zamolodchikov, 'Thermodynamic Bethe ansatz in relativistic models: scaling 3 -state 
Potts and Lee-Yang models', Nucl. Phys. B342 (1990) 695

[27] F.W.J. Olver, Asymptotics and special functions (Academic Press 1974)

[28] D. Fioravanti, F. Ravanini and M. Stanishkov, 'Generalized KdV and quantum inverse scattering description of conformal minimal models', Phys. Lett. B367 (1996) 113, hep-th/9510047,

[29] R.E. Langer, 'On the connection formulas and the solutions of the wave equation', Phys. Rev. 51 (1937) 669 